\title{
STUDIES OF MURINE CORONAVIRUS DI RNA REPLICATION FROM NEGATIVE-STRAND TRANSCRIPTS
}

\author{
S. Banerjee and S. Makino \\ Department of Microbiology, and Institute of Cellular and Molecular Biology \\ The University of Texas at Austin \\ Austin, Texas 78712
}

\section{ABSTRACT}

The positive-strand transcripts as well as negative-strand transcripts of mouse hepatitis virus (MHV) defective interfering (DI) RNA, when introduced into MHV-infected cells, resulted in DI RNA replication and accumulation. The leader sequence of the majority of DI RNAs that accumulated from the expression of negative-strand DI RNA transcripts with no extra non-MHV nucleotides at the 3' end switched to that of helper virus, whereas this leader sequence switching did not occur in most of the positive-strand DI RNAs that accumulated from the expressed negative-strand DI RNA transcripts with extra non-MHV nucleotides at the 3' end. These data demonstrated that the extra 4 nucleotides at the 3'-end of negativestrand DI RNA transcripts affected leader sequence switching on DI RNA, and indicated that the leader switching probably occurred during positive-strand DI RNA synthesis.

\section{INTRODUCTION}

Mouse hepatitis virus (MHV), the prototypic coronavirus, contains a single-strand, positive-sense RNA that is approximately $31 \mathrm{~kb}$ in length (Lee et al., 1991; Lai and Stohlman, 1978). MHV-infected cells generate seven to eight species of virus-specific mRNAs whose sequences comprise a 3'-co-terminal nested set structure (Lai et al., 1981; Leibowitz et al., 1981). At their 5'-ends all of the mRNAs are fused to a 72- to 77-nucleotide-long leader sequence (Lai et al., 1984; Spaan et al., 1983). Genomic sized and subgenomic sized negative-strand RNAs are present in coronavirus-infected cells in amounts that are significantly lower than the amounts of corresponding positive-strand RNAs (Sethna et al., 1989). The subgenomic sized negative-strand RNAs may be template RNAs 
for subgenomic mRNAs (Schaad and Baric, 1994; Sethna et al., 1991; Sawicki and Sawicki, 1990) or they may be dead-end transcription products (Jeong and Makino, 1992).

Complete cDNA clones of coronavirus defective interfering (DI) RNAs under control of the T7 promoter have been used to study various aspects of coronavirus replication. Transfection of in vitro-synthesized positive-strand MHV DI RNA transcripts into MHV-infected cells as well as expression of positive-strand DI RNA transcripts by a recombinant T7 vaccinia virus expression system in MHV-infected cells results in DI RNA replication. The leader sequence of DI RNAs that contain a nine-nucleotide stretch of sequence (UUUAUAAAC) at the junction between the leader and the remaining DI sequence efficiently switches to that of helper virus during replication (Makino and Lai, 1989). This high-frequency leader sequence switching is not observed when the nine-nucleotide are deleted (Makino and Lai, 1989). Similar leader sequence switching is found in other coronavirus DI RNAs (Chang et al., 1996) yet the mechanism of leader switching remains unclear.

MHV DI RNA accumulation occurs in MHV-infected cells when the DI RNA negativestrand transcripts are expressed using a vaccinia virus T7 expression system; DI RNA replication depends on expression of $\mathrm{T} 7$ polymerase and on the presence of the $\mathrm{T} 7$ promoter (Joo et al., 1996). In this study, we examined the leader sequence of DI RNAs that accumulated from expression of two different negative-stranded DI RNA transcripts, and found that the sequence difference at the very 3 ' end of the negative-strand transcripts affected the leader switching of the accumulated DI RNAs. The data shown in this study also indicated that the leader switching in DI RNA probably occurred during positive-strand DI RNA synthesis.

\section{MATERIAL AND METHODS}

\subsection{Viruses and Cells}

The plaque-cloned A59 strain of MHV (Lai et al., 1981) was used as a helper virus. Mouse DBT cells were used for MHV growth and DNA transfection. Recombinant vaccinia virus (VV) vTF7-3 that expresses T7 RNA polymerase (Fuerst et al., 1986) were grown and titered in RK13 cells.

\subsection{DNA Transfection}

DNA transfection into vTF7-3-infected DBT cells was described previously (Joo et al., 1996). A plasmid, pS5A, containing the Chloramphenicol Acetyl Transferase (CAT) gene (Woo et al., 1997) was co-transfected with the DNA.

\subsection{Preparation of Virus-Specific Intracellular RNA}

Viral RNAs from virus-infected cells were extracted as previously described (Makino et al., 1984). In some experiments poly(A) containing RNA was collected by oligo(dT) column chromatography. RNA samples were treated with RNase-free DNase I (Promega) at $37^{\circ} \mathrm{C}$.

\subsection{Northern (RNA) Blotting}

Northern blot analysis using a random-primed probe corresponding to the $0.55 \mathrm{~kb}$ Stu I - Sph I fragment of DE 25 was performed as previously described (Jeong and Mak- 
ino, 1992). For detection of positive-stranded MHV DI-RNA, oligonucleotide 10080 (5' GGCAAGCCGTCCTCTTCTTGGGTATCGGC 3'), which binds at nucleotides 931-960 of DE25 from the 5'-end was 5'-end labeled and used as a probe.

\subsection{Reverse Transcriptase (RT)-PCR}

Positive-strand DI RNA specific-cDNAs were synthesized by incubating intracellular RNA species with oligonucleotide 10120 (5' CTTTAGACAACGCCAGTT 3'), which binds to positive-strand DI RNA at nucleotides 1594 to 1611 nucleotide from 5' end, as described previously (Makino et al., 1988); this oligonucleotide binds at the junction site of domains II and III of the positive strand of DIssE (Makino et al., 1988). RT-PCR products were synthesized by incubating the cDNAs with oligonucleotide 10066 (5'TATAAGAGTGATTGGCGTCCG 3'), which binds to the 5' end of leader sequence (nucleotides 1-21). PCR was carried out at $94^{\circ} \mathrm{C}$ for $5 \mathrm{~min}$ (to inactivate RT), $94^{\circ} \mathrm{C}$ for $30 \mathrm{sec}, 50^{\circ} \mathrm{C}$ for $1 \mathrm{~min} .30 \mathrm{sec}$, and $72^{\circ} \mathrm{C}$ for $1 \mathrm{~min} .30 \mathrm{sec}$, for 30 cycles.

\subsection{Sequence Analysis}

The gel-purified, DI-specific, RT-PCR products were sequenced by cloning the RTPCR products into a TA cloning vector (Invitrogen). To sequence the leader region of these clones oligonucleotide 10258 (5' CTGGCGCCGAATGGACACGTC 3') was used. The oligonucleotide binds to nucleotides $168-188$ of DIssE, from the 5'-end.

\section{RESULTS}

Previously we constructed and characterized two plasmids, pDER and pDER4, to study DI RNA replication from MHV DI RNA negative-strand transcripts (Joo et al., 1996). In pDER a complete cDNA of MHV DI RNA, DIssE, is inserted between the T7 promoter and the hepatitis delta virus ribozyme domain such that negative-strand DI RNA transcripts are synthesized from the T7 promoter. The leader sequence of pDER has 4 repeats of UCUAA at the junction of the leader and body sequences and lacks the nine-nucleotide sequence (UUUAUAAAC) downstream of the UCUAA repeats (on the positive-sense), while MHV-A59 has 2 UCUAA repeats and contains the nine-nucleotide sequence (Makino and Lai, 1989). The leader sequence of pDER also has five substituted nucleotides relative to the MHV leader sequence. The structure of pDER4 is nearly identical to that of pDER, except that pDER4 has four extra nucleotides, AGCT, between the 3'end of the leader sequence and the ribozyme domain; negative-strand transcripts of pDER4 should have the corresponding (UCGA) additional non-MHV sequence at the 3'end (Fig. 1). When the negative-strand transcripts of the DI RNA are expressed in MHVinfected cells by a vaccinia virus $\mathrm{T} 7$ expression system, positive-strand DI RNAs accumulate in the plasmid-transfected cells (Joo et al., 1996). DI RNA replication depends on expression of T7 polymerase and on the presence of the T7 promoter (Joo et al., 1996).

To test whether leader switching occurred in the accumulated DI RNAs from the expressed negative-strand DI RNA transcripts, we examined the leader sequence of accumulated DI RNAs from the cells expressing pDER transcripts and pDER4 transcripts. Intracellular viral RNA was extracted from plasmid-transfected cells at $10 \mathrm{~h}$ p.i. with MHV and incubated with DNase I. We amplified the 5'-region of positive-strand DI RNAs 


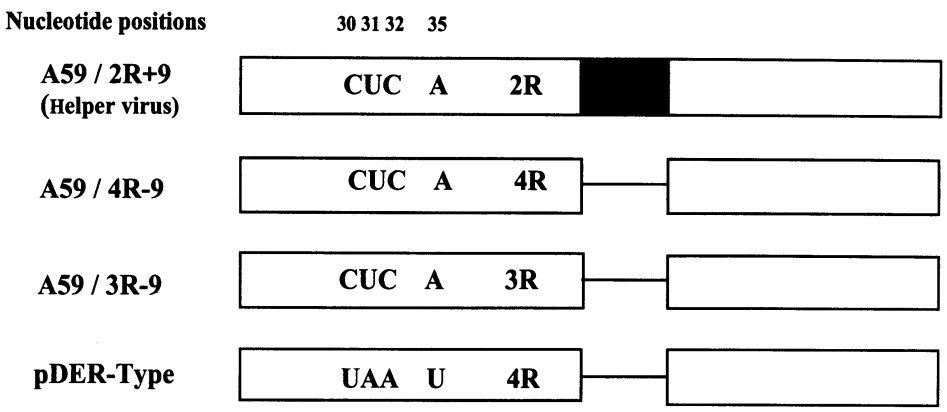

Figure 1. Diagram of the 5 '-end sequences of the DI RNA transcripts in the positive sense. A59/2R+9 represents MHV genomic RNA. The shaded box represents the nine-nucleotide sequence (5' UUUAUAAAC 3'). 2R, 3R and $4 R$ represent two, three and four repeats of UCUAA sequence, respectively. The nine-nucleotide sequence that is deleted is shown as a thin line. The nucleotide sequences which are not denoted share the same sequence as described previously (Makino and Lai, 1989). pDER-type represents both pDER and pDER4. The only difference between pDER and pDER4 is the presence of four extra nucleotides (5' UCGA 3') at the 3'-end of pDER4.

using RT-PCR as described in the Materials and Methods. We detected the expected RTPCR product from DNA-transfected, MHV-infected cells, whereas we did not detect this product in the DNA-transfected, mock-infected cells, or in mock-transfected, MHV-infected cells (data not shown), indicating that the RT-PCR product was indeed derived from positive-strand DI RNAs. The PCR product was purified on an agarose gel and cloned into a plasmid vector. Sequencing analysis of five out of a total of six clones derived from accumulated positive-strand DI RNAs in the pDER-transfected cells had helper virus-specific leader sequence with two repeats of the UCUAA sequence and the nine-nucleotides, while one clone had helper virus-specific leader sequence with four repeats of UCUAA and deletion of the nine-nucleotide sequence. Sequencing analysis of DI RNAs from pDER4-transfected cells showed that five out of nine clones had the pDER4-specific leader sequence. We do not know whether those accumulated DI RNAs maintained the extra 4 nucleotides present at the extreme 3' end of the pDER4 transcripts, because this RTPCR analysis did not identify the very 5' end of the leader sequence. The remaining four clones showed the helper virus-specific leader sequence, yet all of the clones lacked the nine-nucleotide sequence. Half of the clones contained three UCUAA repeats, while the remaining two clones had four UCUAA repeats (Table 1).

The leader switching does not occur after transfection of positive-strand DI RNAs lacking the nine-nucleotides (Makino and Lai, 1989), whereas we showed here that most of the accumulated DI RNAs underwent the leader switching in cells expressing pDER transcripts, which lacked the nine-nucleotides. Furthermore, our data indicated that the presence of 4 extra nucleotides at the very 3 '-end of the negative-strand DI RNA transcripts affected leader switching; it seemed that the extra nucleotides prevented the leader switching.

\section{DISCUSSION}

The present data provided further information about the leader switching mechanism. The leader switching does not occur when positive-strand DI RNA transcripts lacking the nine-nucleotide sequence are transfected into MHV-infected cells (Makino and 
Table 1. Leader sequences of cloned PCR products of DI RNAs from MHV-infected pDER-expressing cells, and from MHV-infected pDER4-expressing cells

\begin{tabular}{lcccc}
\hline & \multicolumn{4}{c}{ Leader type $^{\mathrm{a}}$} \\
\cline { 2 - 5 } Transfected clones $^{\mathrm{b}}$ & A59/2R+9 & A59/3R-9 & A59/4R-9 & pDER-type \\
\hline${\text { pDER }+ \text { pS5 } 5 \mathrm{~A}^{\mathrm{c}}}^{\mathrm{d}}$ & 5 & - & 1 & - \\
pDER4 + pS5A & - & 2 & 2 & 5 \\
\hline
\end{tabular}

\footnotetext{
${ }^{a}$ Leader sequences as described in Figure 1.

${ }^{b}$ Positive-strand DI RNA-specific RT-PCR products were cloned into TA cloning vector. Sequence analysis of leader regions of the cloned PCR products was performed.

${ }^{c}$ Five clones out of six had helper virus leader sequences. One clone had helper virus type leader with four UCUAA repeats and lacking the nine-nucleotide sequence.

dOut of nine clones sequenced, two clones had A59/3R-9 leader, two had A59/4R-9 leader and five retained the pDER-type leader.
}

Lai, 1989). Accordingly, accumulated negative-stranded DI RNAs also most likely lack the nine-nucleotide sequence. Most MHV negative-strand RNAs appear to exist in doublestrand (ds) forms (Sawicki and Sawicki, 1986), therefore, the majority of negative-strand DI RNAs and some positive-strand DI RNA probably formed ds RNAs. We assume that efficient positive-strand RNA synthesis occurs from those ds RNAs. If the positive-strand DI RNA molecules that were synthesized from the expressed pDER transcripts are faithful copies of the pDER transcripts, then they should have lacked the nine-nucleotides. If this is the case, the ds RNAs made of newly synthesized positive-strand DI RNA and expressed negative-strand pDER DI RNA transcripts are structurally very similar to the ds RNAs that are produced after transfection of positive-strand DI RNA transcripts lacking the nine-nucleotides. Accordingly, one might expect that the structure of nascent positivestrand DI RNAs from both of those ds DI RNAs is the same. However, positive-strand DI RNAs accumulated in the cells expressing pDER transcripts contain helper virus-derived leader sequence, whereas those accumulated after transfection of the positive-strand DI RNA transcripts do not undergo leader switching. Therefore, we speculate that initial positive-strand DI RNA molecules synthesized from the expressed pDER transcripts were not faithful copies of the pDER. It is more likely that the helper virus-derived RNA(s) containing the leader sequence was used as a primer for the initiation of positive-strand DI RNA (Makino and Lai, 1989); this would result in leader switching on DI RNAs after expression of pDER transcripts. We further speculate that if the template RNAs exist as ds RNAs, this primer-dependent initiation of positive-strand DI RNA does not occur. This speculation may explain why those DI RNAs that accumulate after transfection of the positive-strand DI RNAs do not undergo leader switching. The ds DI RNAs produced after transfection of the positive-strand DI RNA transcripts consist of in-put DI RNA transcripts and faithful copies of the negative-strand DI RNAs. Subsequent positive-strand DI RNA synthesis does not require the helper virus-derived RNA containing the leader sequence as a primer, and the accumulated DI RNAs maintain the original leader sequence.

The leader switching did not occur in the majority of DI RNAs that accumulated in the cells expressing pDER4 transcripts, indicating that the presence of the extra 4 nucleotides at the very 3 '-end of pDER4 transcripts was crucial for the leader switching. Why did most of the positive-strand DI RNAs that accumulated from the expressed pDER4 transcripts not undergo leader switching? The extra 4 nucleotides at the very 3' end of pDER4 transcripts and sequence immediately upstream may form a specific secondary structure, which may facilitate the initiation of positive-strand DI RNA synthesis. If the 
very 3 ' end negative-strand transcript lacks extra nucleotides, i.e., pDER transcripts, the very 3'-end of the expressed transcripts may not form the secondary structure that is optimal for the initiation of positive-strand DI RNA synthesis. If nascent positive-strand DI RNAs are not initiated from the very 3'-end of the negative-strand DI RNA transcripts, then MHV replication mechanism may use helper virus-derived leader sequence as a primer for the initiation of positive-strand DI RNA synthesis.

\section{ACKNOWLEDGMENTS}

This work was supported by Public Health Service grants AI29984 and AI32591 from the National Institutes of Health.

\section{REFERENCES}

Chang, R.-Y., Krishnan, R. and Brian, David, 1996, The UCUAAAC promoter motif is not required for high frequency leader recombination in bovine coronavirus defective interfering RNA, J. Virol. 70:2720-2729.

Fuerst, T.R., Niles, E.G., Studier, F.W., and Moss, B., 1986, Eukaryotic transient-expression system based on recombination vaccinia virus that synthesizes bacteriophage T7 polymerase, Proc. Natl. Acad. Sci. USA 83:8122-8126.

Jeong, Y.S., and Makino, S., 1992, Mechanism of coronavirus transcription:duration of primary transcription initiation activity and effect of subgenomic RNA transcription on RNA replication, J. Virol. 66:3339-3346.

Lai, M.M.C., and Stohlman, S.A., 1978, RNA of mouse hepatitis virus, J. Virol.. 26:236-242.

Lai, M.M.C., Baric, R.S., Brayton, P.R. and Stohlman, S.A., 1984, Characterization of leader RNA sequences on the virion and mRNA of mouse hepatitis virus, a cytoplasmic virus, Proc. Natl. Acad. Sci. USA 81:3626-3630.

Lai, M.M.C., Brayton, P.R., Armen, R.C., Patton, C.D., Pugh, C., and Stohlman, S.A., 1981, Mouse hepatitis virus A59: mRNA structure and genetic localization of the sequence divergence from hepatotropic strain MHV3, J. Virol. 39:823-834.

Lee, H.-J., Shieh, C.-K., Gorbalenya, A.E., Eugene, E.V., La Monica, N., Tuler, J., Bagdzhadzhyan, A., and Lai, M.M.C., 1991, The complete sequence (22 kilobases) of murine coronavirus genel encoding the putative proteases and RNA polymerase, Virology 180:567-582.

Leibowitz, J.L., Wilhelmsen, K.C., and Bond, C.W., 1981, The virus-specific intracellular RNA species of two murine coronaviruses: MHV-A59 and MHV-JHM, Virology 114:39-51.

Makino, S., and Lai, M.M.C., 1989, High frequency leader sequence switching during coronavirus defective interfering RNA replication, J. Virol. 63:5285-5292.

Makino, S., Taguchi, F., Hirano, N., and Fujiwara, K., 1984, Analysis of genomic and intracellular viral RNAs of small plaque mutants of mouse hepatitis virus, JHM strain, Virology 139:138-151.

Makino, S., Shieh, C.-K., Soe, L.H., Baker, S., and Lai, M.M.C., 1988, Primary structure and translation of a defective interfering RNA of murine coronavirus, Virology 166:550-560.

Sawicki, S.G., and Sawicki, D.L., 1990, Coronavirus transcription: subgenomic mouse hepatitis virus replicative intermediates function in RNA synthesis, J. Virol. 64:1050-1056.

Sawicki, S.G., and Sawicki, D.L., 1986, Coronavirus minus-strand mRNA synthesis and effect of cyclohexamide on coronavirus RNA synthesis, J. Virol. 57:328-334.

Schaad, M.C., and Baric, R.S., 1994, Genetics of mouse hepatitis virus transcription: evidence that subgenomic negative strands are functional templates, $J$. Virol. 68:8169-8179.

Sethna, P.B., Hofmann, M.A., and Brian, D.A., 1991, Minus-strand copies of replicating coronavirus mRNA contain antileaders, J. Virol. 65:320-325.

Sethna, P.B., Hung, S.-L., and Brian, D.A., 1989, Coronavirus subgenomic minus-strand RNAs and the potential for mRNA replicons, Proc. Natl. Acad. Sci. USA 86:5626-5630.

Spaan, W., Delius, H., Skinner, M., Armstrong, J., Rottier, P., Smeekens, S., van der Zeijst, B.A.M., and Siddell, S.G., 1983, Coronavirus mRNA synthesis involves fusion of non- contiguous sequences, EMBO J. 2:1939-1944.

Woo, K., Joo, M., Narayanan, K., Kim, K.H., and Makino, S., 1997, Murine coronavirus packaging signal confers packaging to nonviral RNA, J. Virol. 71:824-827. 